\title{
Oil Leakage Detection Using Various Thicknesses Technique
}

\author{
${ }^{1}$ Ayad Z. Mohammed, ${ }^{2}$ Bushra R.Mahdi, ${ }^{3}$ Aseel H.Ajlan \\ ${ }^{1}$ Ayad Z. Mohammed, Asst. Prof., Optoelectronics Engineering Department, University of Technology, Baghdad, Iraq. \\ ${ }^{2}$ BushraR.Mahdi, Dr.,Ministry of Science and Technology, Baghdad, Iraq. \\ ${ }^{3}$ AseelH. Ajlan, M.ScStudent, Optoelectronics Engineering Department, University of Technology, Baghdad, Iraq.
}

Received 2017; Accepted 2017; Available online 2017

\author{
Address For Correspondence: \\ AseelH. Ajlan, University of Technology, Optoelectronics Engineering Department, engineering collage, Baghdad, Iraq. \\ E-mail: Aseelhussain1987@gmail.com
}

Copyright (C) 2017 by authors and American-Eurasian Network for ScientificInformation (AENSI Publication). This work is licensed under the Creative Commons Attribution International License (CC BY).

http://creativecommons.org/licenses/by/4.0/

\begin{abstract}
Solid core PCF (LMA-10) is dependent which based on the principle of the Mach Zender interference to detect the leakage of oil bearing pipes. The basic structure of the sensor includes small pieces with different lengths of the solid core photonic Crystal fiber spliced with the using traditional single mode fibers (SMF-25) by molten splice in both sides. The air holes at the fusion zone are completely collapsed, allowing the core cladding patterns to irritate and recombine the core cladding.The pipes different thickness causes wavelengths shifting and variation in the sensor intensity. The immediate phenomenon caused by erosion is the pipeline wall thickness decreasing. The pipeline wall thickness decreasing could be reflected by the obtained hoop strain dep ending on the theory principle that hoop strain is proportion conversely with pipeline wall thickness. The PVC and the steel pipe patterns are utilized to simulate the pipeline with uniform erosion. In this paper a sensor of three different length is used $(2,2.5,3) \mathrm{cm}$ and examined to know the most sensitive sensor to pipe thickness change <Leak> at temperature $\left(36^{\circ} \mathrm{C}\right)$ where the leakage is known by a change in the external intensity or radiation deviation to the red shift area, and the calculation of the stress generated by cracks which is inversely proportional to the pipe wall thickness. The experimental results show that the sensor of the highest sensitivity is the second sensor $2.5 \mathrm{~cm}$ where it is about 0.31 for metal pipe and 0.25 for polymer pipe.
\end{abstract}

KEYWORDS: Optical fiber, Photonic Crystal Fiber PCF, Oil leakage detection, Wall thickness.

\section{INTRODUCTION}

Two type of optical fiber are excited relying upon modes number and cylindrical structure: single mode fiber SMF which consist of single mode and it differs in core diameter from the multimode and it has small core diameter equal to (5 - 10 microns) while multimode fiber MMF which consist of multiple mode and its core diameter is larger than single equal to (50 or 62.5 microns) [1].

Many sorts of optical fiber sensors have been considered to gauge various parameters. More than 70 various parameters could be measured or checked, for example, the refractive list, revolution, vibration, temperature, attractive fields, weight, strain, acoustics, stickiness, increasing speed, etc. Optical fiber sensors have many benefits, for example, invulnerable to electromagnetic interference, lightweight, high sensitivity, and additional resistance from cruel environmental, remote detecting capacity [2].

Photonic Crystal Fiber PCF which is a new sort of optical fiber is shown by Russell. It can beat numerous impediment of traditional optical fiber, PCFs are special class of optical fiber can be produced using single material (silica), its geometry is highlighting by special arrangement of air-hole that running along whole length of fiber, by consolidating the properties of both optical fibers and photonic crystals [3]. 


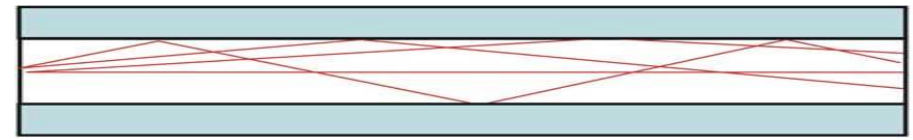

Multimode, Step-index

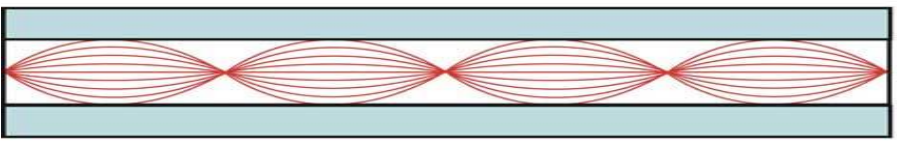

Multimode, Graded Index

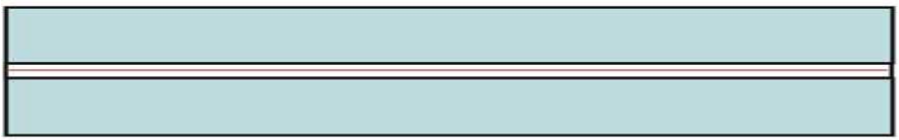

Singlemode
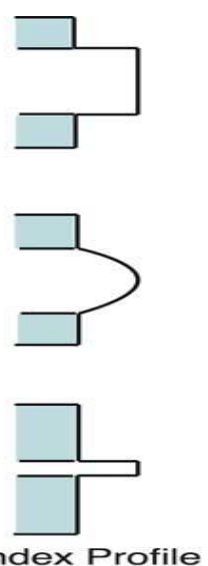

Index Profile

Fig. 1: Optical FiberTypes

\section{Methodology:}

LMA-10 PCFS is utilized in this part of work, different lengths of the utilized PCF $(2,2.5,3) \mathrm{cm}$, are joined by combination splice machine Fujikura (FSM 60). Collapsing method is applied in this sensor amid the sensor creation, so the sensor transmission range checked with $850 \mathrm{~nm}$ laser diode source and OSA as Optical Spectrum Analyzer. The sensor structure is demonstrated in figure (2).

The pipes different thickness causes wavelengths shifting and variation in the sensor intensity. The immediate phenomenon caused by erosion is the pipeline wall thickness decreasing. The pipeline wall thickness decreasing could be reflected by the obtained hoop strain depending on the theory principle that hoop strain is proportion conversely with pipeline wall thickness. The PVC and the steel pipe patterns are utilized to simulate the pipeline with uniform erosion [4-8].
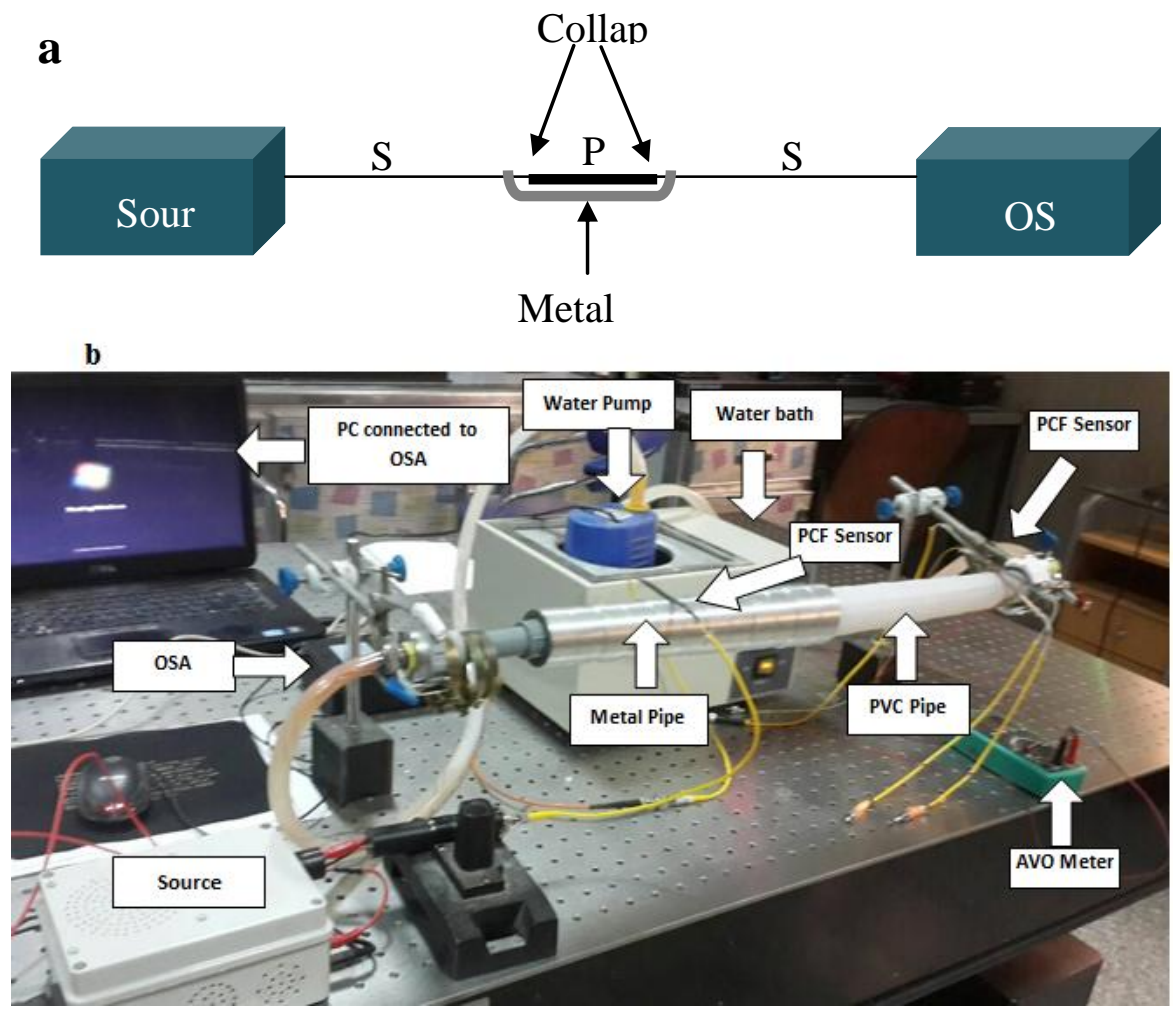

Fig. 2:a) Schematic of proposed sensors

b) Experiment setup

In this work the ordinary single mode fiber (SMF-28), has been utilized with photonic crystal fiber, which has a strong unadulterated encompassing by four coatings of air holes masterminded in a hexagonal model as appeared in figure (3).

With the following physical properties: 
- $\quad 10.1 \pm 0.5 \mu \mathrm{m}$ core diameter.

- $125 \pm 2 \mu \mathrm{m}$ outer cladding diameter across.

- $\quad 3.1 \mu \mathrm{m}$ air gap width.

- $6.6 \mu \mathrm{m}$ pitch.

By utilizing mechanical combination splice the both sides of PCF and SMF are combined, the combination power and time have been changed; collapse region used in this work is equal to $250-300 \mu \mathrm{m}$, as appeared in figure (4). The air gaps are totally collapse the core mode spreads into the first collapsed region this situation for the first splice region; its mode field width is expanded, causing excitation of the core and cladding modes.Then associated after spreading a separation of $\mathrm{L}_{\mathrm{PCF}}$ (PCF length) second collapsed region[9-12].

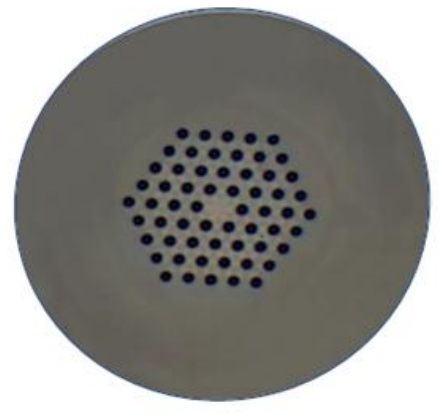

Fig. 3: microscope image of cross section of theLMA-10 PCF used for the experiment, when the magnification power of microscope $(20 \mathrm{X})$

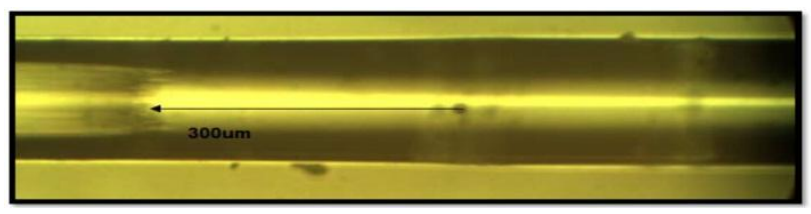

Fig. 4: Microscope image of collapse zone splicing between PCF LMA 10 on the right and SMF on the left the collapsed length is $300 \mathrm{um}$

\section{RESULT AND DISSCUSION}

1. The PCF LMA-10 of $2 \mathrm{~cm}$ length for $\mathrm{S} 1$ and $2.5 \mathrm{~cm}$ length for $\mathrm{S} 2$ and $3 \mathrm{~cm}$ length for $\mathrm{S} 3$ are joined with SMF - 28 from both sides.

2. The two collapsed regions are physically presented by varying the arc duration time and power.

3. The collapsed regions length is around $300 \mu \mathrm{m}$.

4. The PCF sensor is covered by a metal holder to enhance the thermal conductivity.

5. A laser diode $850 \mathrm{~nm}$ source is utilized. The light passing through SMF to PCF, then the sensor is connected to one of the utilized pipes (polymer or steel) with different temperature, thickness and stream - rate pumping oil.

6. $4.5 \mathrm{~mm}$ diameter stainless steel pipe is utilized and with $30 \mathrm{~cm}$ length.

7. Pipe wall thickness is re framed to have different thickness, beginning with $d_{1}=7 \mathrm{~mm}$ and consummation with $\mathrm{d}_{12}=1.5 \mathrm{~mm}$ with $0.5 \mathrm{~mm}$ steps by utilizing a CN machine.

8. For heating the fluids a water path is utilized.

9. The fluid temperature is held constant at $36^{\circ} \mathrm{c}$ which encompassing temperature.

10. The different sensors lengths are analyzed on all pipe zones, which are connected to the pipes wall.

11. The sensor output is associated with the OSA and the spectrum output yield wavelengths shifting or intensity losses.

12. Power and intensity measurements are acquired from OSA and power meter for every thickness.

13. The strain is computed on each pipes wall thickness.

The metal pipe results at different thickness at ambient temperature are shown in figure (5). The figure shows the transmission spectrum for $\mathrm{S} 1$ of $2 \mathrm{~cm}$ length at different thickness from (d1-d12) at ambient temperature for metal pipe. 


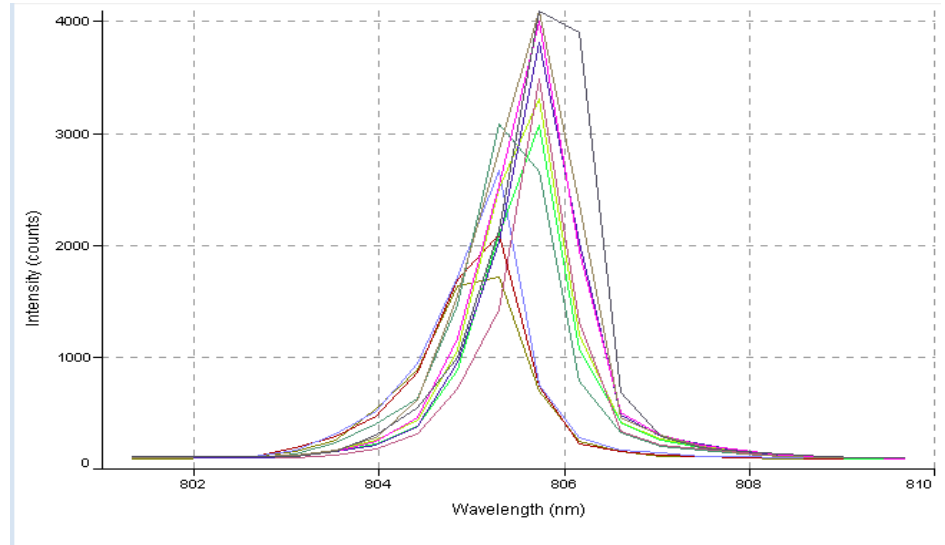

Fig. 5: Transmission spectra of $2 \mathrm{~cm}$ LMA-10 PCF interferometer for sensing leak at different wall thickness for metal pipe

Figure(6) shows the red shifting when the thickness is increased. The shifting is caused by the strain that is proportional inversely with the wall thickness.

Figure(7) shows the relation between thickness and intensity whereas the increase of wall thickness leads to increase in intensity. As a result, the sensitivity of the sensor can be calculated, and it's equal to (0.19).

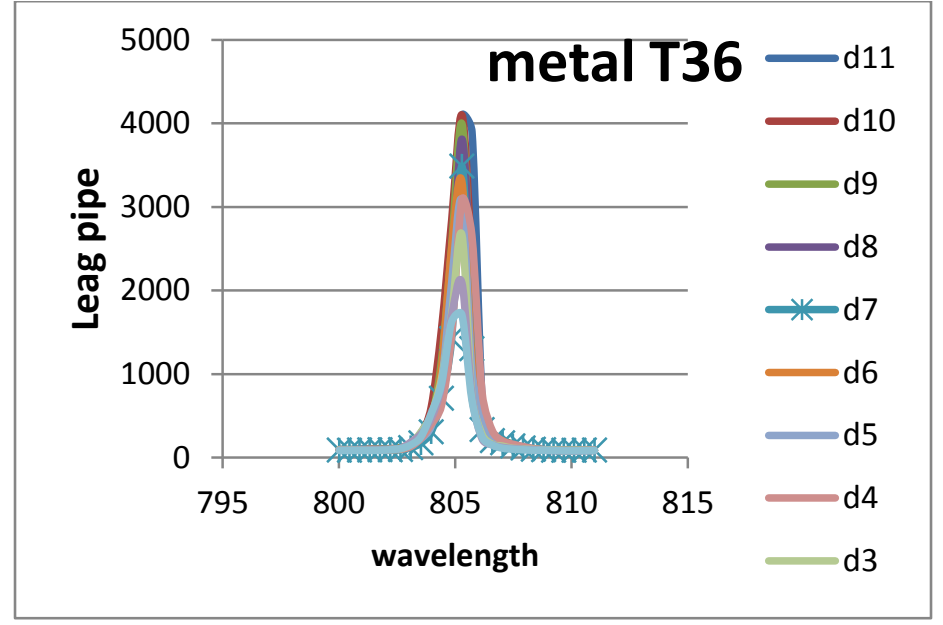

Fig. 6: Transmission spectra of 2 cmLMA-10 PCF interferometer for sensing leak at different wall thickness for metal pipe $\mathrm{S} 1$

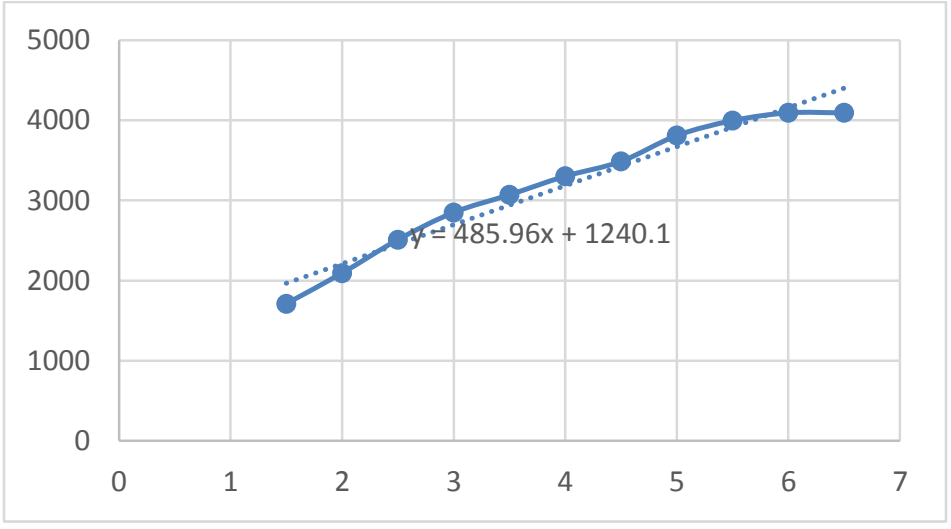

Fig.7: The relationship between intensity and wall thickness for $2 \mathrm{~cm}$ LMA-10 PCF sensor1 of metal pipe $($ sensitivity $=0.19 \mathrm{~nm} / \mathrm{RIU})$

Figure (7) illustrates the relation between wall thickness and intensity of laser beam, which passes through $\mathrm{S} 2$ of polymer pipe whereas the calculated sensitivity from this figure is equal to $(0.25 \mathrm{~nm} / \mathrm{RIU})$. 
Figure (8) shows the measured transmission spectrum for $3 \mathrm{~cm}$ LMA-10PCF S2 at different thickness from (d1-d12) at ambient temperature for metal pipe.

Figure (9) shows the red shifting when the wall thickness of metal pipe is increased. The maximum shifting at $\mathrm{d} 12$ is equal to $7 \mathrm{~mm}$.

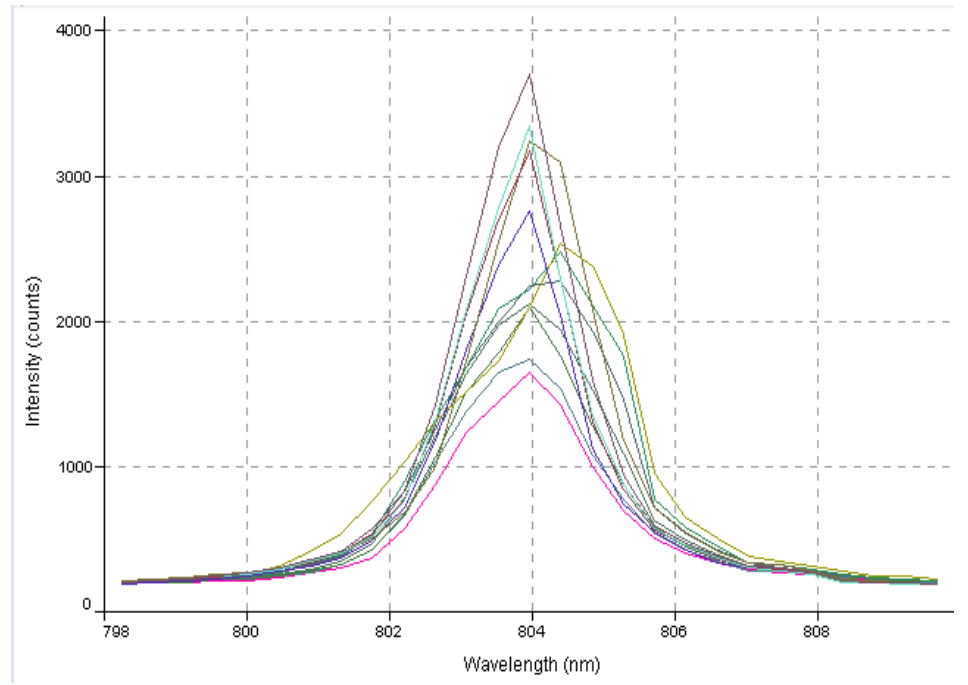

Fig. 8: Transmission spectra of $3 \mathrm{~cm}$ LMA-10 PCF interferometer for sensing leak at different wall thickness for metal pipe S3

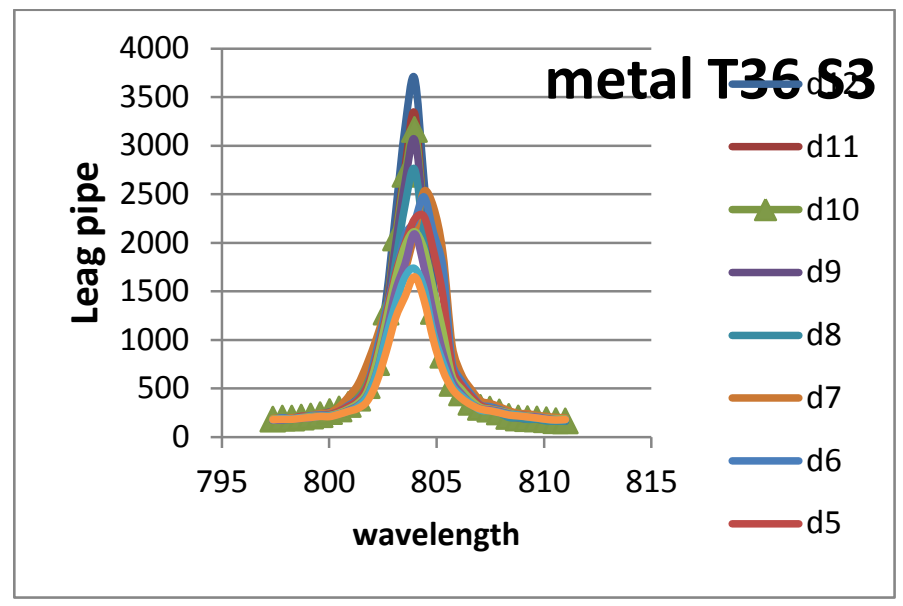

Fig. 9: Transmission spectra of $3 \mathrm{~cm}$ LMA-10 PCF interferometer for sensing leak at different wall thickness for metal pipe S3

\section{Conclusion:}

A completedetectionfor the oil pipes leakageusingvariousthickness methodis utilized and performed successfully. The results of this work showed that the wall thickness for the various thicknesses technique is ejective proportional with the external intensity and the second sensor of length $2.5 \mathrm{~cm}$ has the best sensitivity for this technique and it is about 0.31 for metal and 0.25 for polymer and this is the best value can be obtained.

Also utilizing Optical Time Domain Reflectometer OTDR is a good idea for leakage sensing.

\section{REFERANCES}

1. FedorMitschke, 2009. "fiber optics", Rostock University, Germany, 18055.

2. Ning, Y.N. and B.T. Meggitt, 1998. "Fiber Bragg grating sensors: signal processing aspects", Optical fiber sensor technology, Springer US, pp: 381-417.

3. Fidanboylu, K.A. and H.S. Efendioglu, 2009. "Fiber optic sensors and their applications", 5th International Advanced Technologies Symposium (IATS'09), 6. 
4. Sharma, Nidhi, Neetu Rajawat and Kavita Agrawal, "Photonic Crystal Fiber Characteristics Benefits Numerous Applications." International Journal of Scientific and Engeneering Research, 6: 28-33.

5. Schindler, Paul M., 1995. Corrosion detection using metal coatings on fiber optic sensors. Diss. Virginia Polytechnic Institute and State University.

6. Tennyson, Rod C., W. Don Morison, and Tom Miesner. 2006. "FOS types."

7. Li, Mo and Yunhao Liu, 2007. "Underground structure monitoring with wireless sensor networks." Proceedings of the 6th international conference on Information processing in sensor networks. ACM.

8. Jha, R., J. Villatoro, G. Badenes, 2008. Ultrastable in reflection photonic crystal fiber modal interferometer for accurate refractive index sensing. Appl. Phys. Lett., 93: 191106.

9. Nikles, Marc, 2009. "Long-distance fiber optic sensing solutions for pipeline leakage, intrusion and ground movement detection." Proc. SPIE., 7316: 1.

10. Mahdi, B.R., H.D. AL-Attabi, S.D. Salman, 2016. “Manufacture of fiber optic sensors to measure the PH water" Current Trends in Natural Sciences, 5(9): 55-61.

11. Ayad Zwayen M Al-Juboori, 2013. " Rare earth $(\mathrm{Sm}+3$ and $\mathrm{Dy}+3)$ doped gadolinium oxide Nanomaterial's for Fluorescence thermometry" Phys. Scr., T157 014004 (5pp).

12. Ayad, Z.,Mohammed, Bushra R. Mahdi, Lena K. Humdi, 2017." Photonic crystal fiber magnetic field sensor based on Ampere Force" Sci.Int.(Lahore), 29(1): 99-102. 